\title{
High-fill factor micro-mirror array for multi object spectroscopy
}

\author{
Severin Waldis ${ }^{a}$, Pierre-Andre Clerc ${ }^{a}$, Frederic Zamkotsian ${ }^{b}$, Michael Zickar $^{a}$, Wilfried Noell ${ }^{a}$, \\ Nico de Rooij ${ }^{a}$ \\ ${ }^{a}$ IMT, University of Neuchatel, Jaquet Droz 1, CH-2007 Neuchatel, Switzerland \\ ${ }^{b}$ Laboratoire d'Astrophysique de Marseille, 2 place Leverrier, F-13248 Marseille Cedex 4, \\ France
}

\begin{abstract}
Programmable multi-slit masks are required for next generation Multi-Object Spectrograph (MOS) for space as well as for ground-based instruments. A promising solution is the use of MOEMS devices such as micromirror arrays (MMA) or micro-shutter arrays (MSA), which both allow the remote control of the multi-slit configuration in real time. In the present work we developed and microfabricated a novel micro mirror array suited for this application. The requirements are: high contrast, optically flat $(\lambda / 20)$ mirrors in operation, high fill factor, uniform tilt angle over the whole array and low actuation voltage. In order to fulfill these requirements we use a combination of bulk and surface micromachining in silicon. The mirrors are actuated electrostatically by a separate electrode chip. The mirrors are defined by deep reactive ion etching in the $10 \mu \mathrm{m}$ thick device layer of a silicon-on-insulator (SOI) wafer, whereas the suspension of the mirrors is defined by a patterned poly-silicon layer hidden on the backside of the mirrors. The mirror size is $100 \times 200 \mu \mathrm{m}^{2}$ and the dimensions of a typical cantilever suspension are $100 \times 5 \times 0.6 \mu \mathrm{m}^{3}$. On a separate SOI wafer the electrodes and the spacers are processed by using a self aligned delayed mask process. The first results on the mirror chips show that the micromirrors can easily achieve the desired mechanical tilt angle of more than $20^{\circ}$ associated with a good surface quality, which is necessary for a high contrast spectroscopy.
\end{abstract}

Keywords: Micromirror array, multi object spectroscopy, MOEMS, DRIE

\section{INTRODUCTION AND BACKGROUND}

Major telescopes around the world are equipped with Multi-Object Spectrographs (MOS) in order to record simultaneously several hundred spectra during a single observation run. Next generation MOS for space like the Near Infrared Multi-Object Spectrograph (NIRSpec) for the James Webb Space Telescope (JWST) require a programmable multi-slit mask. ${ }^{1}$ Conventional masks or complex fiber-optics-based mechanisms are impracticable in space. The programmable multi-slit mask requires remote control of the multi-slit configuration in real time. A promising solution is the use of MOEMS devices such as micro-mirror arrays (MMAs) or micro-shutter arrays (MSAs). Next generation ground-based MOS will also benefit from these developments. MOEMS are based on mature silicon micro-system technology. Their main advantages are their compactness, scalability, and specific task customization using elementary building blocks. While the development of this technology is expensive, these systems are easily replicable and the price of the components is decreasing when their production volume is increased. The typical size of these micro-elements is in the order of $100 \mu \mathrm{m}$, and MMAs are designed for generating reflecting slits, while MSAs generate transmissive slits. MSA has been selected to be the multislit device for NIRSpec and is under development at the NASA's Goddard Space Flight Center. They use a combination of magnetic force for shutter opening, and electrostatic force for shutter latching in the open position. ${ }^{2}$ By placing the programmable slit mask in the focal plane of the telescope, the light from selected objects is directed toward the spectrograph, while the light from other objects and from the sky background is

Further author information:

S.W.: E-mail: severin.waldis@unine.ch, Telephone: +4132720 5571

F.Z.: E-mail: frederic.zamkotsian@oamp.fr, Telephone: + 33495044151 

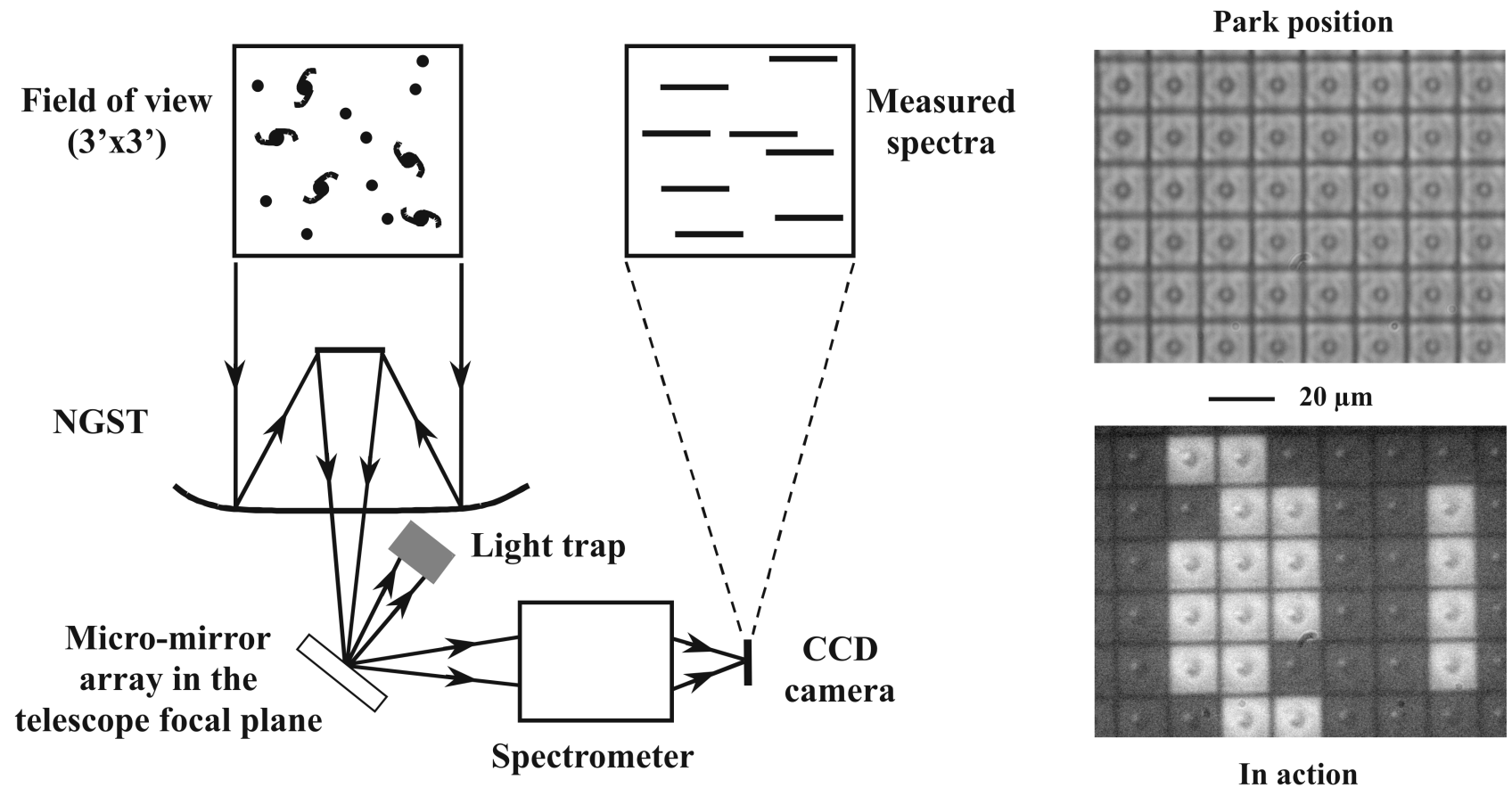

In action

Figure 1. Principle of a Multi-Object Spectrograph with a Micro-Mirror Array for JWST-NIRSpec.

blocked. For the case of a MMA as a programmable slit mask, the MOEMS-based MOS concept is shown in Figure 1.

Using a MMA (here a commercial TI-DMD), any required slit configuration might be obtained with the capability to match point sources or extended objects (Figure 1). In the park position, i.e., without driving voltage applied, the micro-mirrors are undeflected, parallel with the substrate. In action, the micro-mirrors in the 'ON' position direct the light toward the spectrograph and appear bright, while the micro-mirrors in the 'OFF' position are dark. The circle located at the center of the micro-mirrors is the attachment post of the mirror, on top of the tiltable electrodes, made with the mirror material.

In Laboratoire d'Astrophysique de Marseille, we have developed over several years different tools for the modeling and the characterization of these MOEMS-based slit masks, during the design studies on JWSTNIRSpec. Our models, based on Fourier theory, address two key parameters for the MOS performance: spectral photometric variation (SPV) and contrast. The SPV is the unpredictable photometric variation due to the random repartition of the sources on the slit mask. The SPV requirement is generally $<10 \%$, but as SPV is strongly dependent on the object position and wavelength, the required value cannot be reached. We have proposed a dithering strategy which is able to solve this problem. ${ }^{1}$ Contrast is defined as the total amount of non-selected flux of light passing through the multi-slit device. To avoid spoiler sources (bright stars or galaxies within the instrument field of view) and background to pollute spectra, its value has to be as high as possible. According to the density of objects (stars and galaxies) in the field of view and their magnitude, a contrast requirement of 3000 has been established. We have also developed a characterization bench to measure these parameters. Contrast measurement has been carried out on the MMA fabricated by Texas Instrument, in order to simulate the actual MOEMS device for NIRSpec. Effective contrasts of around 500 have been measured for an $\mathrm{ON}-\mathrm{OFF}$ angle of $10^{\circ}$; this value is exceeding 3000 when the ON-OFF angle is $20^{\circ}$. Effects of object position on the micro-mirrors have been revealed. ${ }^{3}$ The effective contrast is the contrast to be considered in an instrument, i.e. the local contrast values integrated on a detector pixel size. Then, in order to reach the requirement, a tilting angle of $20^{\circ}$ is mandatory. Additional parameters such as the size of the source, the wavelength, and the input and output pupil size have been also analyzed.

OPTICON is the network gathering the research efforts of the European astronomical community; a Joint 
a)

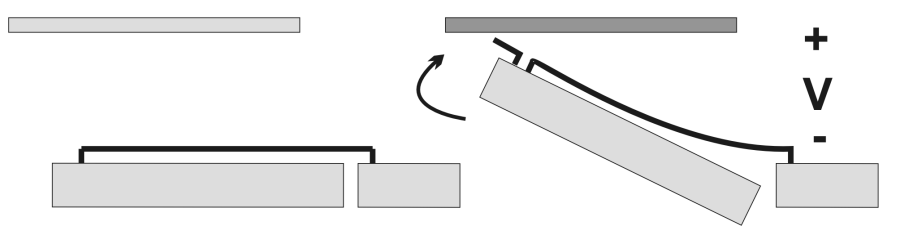

b)

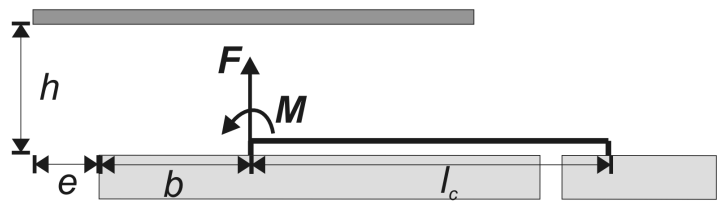

Figure 2. a) Basic operation of the micromirror. The device consists of a suspended mirror and an electrode. By applying a voltage between electrode and mirror the mirror tilts towards the electrode. b) Suspension model. The electrostatic forces acting on the mirror are replaced by a resulting force and moment acting on the endpoint of the suspension cantilever.

Research Activity (JRA) has been set on Smart Focal Planes for developing components to be used in the focal plane of the telescope for selecting or re-arranging the light of the astronomical objects. Within the framework of this JRA, micro-mirrors have been selected in order to get a first demonstrator of an European MOEMS-based slit mask. The Institute of Microtechnology (IMT) of University of Neuchatel (Switzerland) is among the major European institutes in the MEMS field. The laboratory is involved in numerous developments of sensors as well as telecom devices. ${ }^{4}$ Based on our expertise, IMT and LAM engaged a collaboration for developing micro-optical devices suited for astronomical instrumentation.

Based on our simulations and measurements, we have fixed several parameters: In a first approach we set one micromirror per astronomical object, which corresponds to the baseline for NIRSpec. It is essential for this instrument to achieve a high optical contrast of at least 3000:1. The tilted micromirror is used for the ' $O N$ ' position and the rest position is considered as 'OFF'. Hence the amount of parasitic light can be drastically minimized that comes from reflections and scattering of the frame surrounding the micromirrors and of the underneath electrodes. A usable tilting angle must exceed $20^{\circ}$ and the tilt angle must be uniform over the whole array. The surface quality of the micromirrors has to be better than $\lambda / 20$ during operation and throughout a large temperature range. The fill factor of more than $90 \%$ is essential. One micromirror element has to be at least $100 \times 200 \mu \mathrm{m}^{2}$, in order to correspond with the plate scale of $8 \mathrm{~m}$-class telescopes as well as future extremely large telescopes (ELT's). Preferably, the driving voltage for tilting remains below 100V. The micro mirror array has to work at cryogenic temperatures.

We present in this paper the basic concept of the developed device, its analytical modeling as well as finite element method simulation and the fabrication process. Finally we report on the preliminary electromechanical and optical characterization of the first samples.

\section{CONCEPTION AND MODELING}

\subsection{Basic concept}

The basic concept of the device and its operation is showed in Figure 2. A single cell of the device consists of a mirror, which is suspended to a supporting frame by a flexible beam, an electrode and a spacer element that provides a constant gap between mirror and electrode. By applying a voltage between the mirror (or, the frame, assuming the suspension is conductive) and the electrode causes the mirror to tilt, whereas the tilting movement depends upon the geometry of the suspension and of the electrode, and the applied voltage. Physically, the device is realized on two different chips: the mirror chip and the electrode chip. The latter contains the spacer elements. In order to have mirrors with a planarity better than $\lambda / 20$, thick mirrors are used. With a mirror size of $100 \times 200 \mu \mathrm{m}^{2}$, a thickness of $10 \mu \mathrm{m}$ is supposed to be sufficient in order to keep the mirror flat during actuation. The suspension, either a flexion or torsion beam, is situated on the backside of the mirror. This hidden suspension beam configuration leads to a higher fill-factor than lateral suspension beams. In addition, as the suspension is covered by the mirror (except for the small gap between mirror and frame), we have no stray light coming from the bent beams, which means less degradation of the contrast. The contrast value depends upon the tilt-angle of the mirror, that is the angle between the 'OFF' and the 'ON' state of one mirror. The degradation of the contrast is usually due to the stray light originating from the mirror edges, supporting frame, 


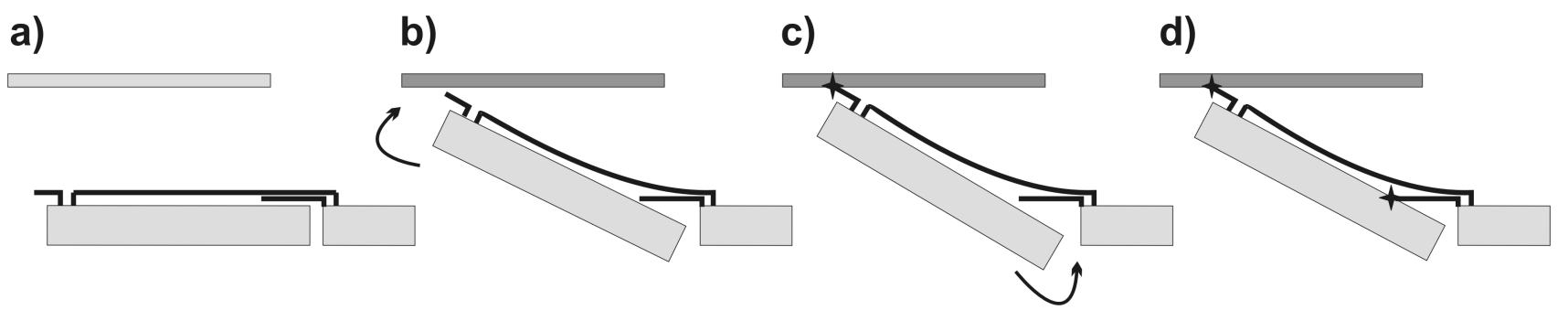

Figure 3. Schematic view of the pull-back and electrostatic latching mechanism: Due to the electrostatic force the mirror rotates upwards (b) until the first landing pad, which is attached to the mirror, hits the electrode (c). Then the mirror starts rotating in the inverse direction until it hits the second landing post, which is attached to the mirror frame (d), and remains electrostatically fixed in this position. Thus the final tilting angle is practically independent on the actuation voltage and relies merely on a homogeneous spacing between the mirror and electrode chips.

suspension and backscattered light from the electrode. As the suspension is hidden by the mirror and the gap-size between mirror and frame is small, the degradation of the contrast is mainly due to the rounding of the mirror edges, surface roughness of the mirror and of the frame. The tilt angle is a function of the gap between the electrode and the mirror and the geometry of the suspension. With a scheduled gap height of $35 \mu \mathrm{m}$, tilting angles between $15^{\circ}$ and $24^{\circ}$ can be achieved. A tilt angle of $20^{\circ}$ should yield a contrast better than 3000:1, as exhibited in section 1. A system of landing posts on the mirror and on the frame has been developed to assure a precise and constant tilt-angle. This concept is shown in Figure 3: Once the mirror (i.e. the landing post located on the mirror) touches the electrode, it will not stop moving but start to turn into the opposite direction around this new rotation axis. That is, the tilting angle tends to decrease once the mirror has landed. This is due to a non-zero (and opposite to the mirror tilting action) torque around the point where the landing post is attached to the mirror. The reverse turning movement is stopped at a well-defined tilt-angle by the landing post attached to the frame surrounding the mirror. The mirror is now electrostatically clamped in a position defined by the geometry of the landing posts and the gap between electrode and mirror.

The mirror and electrode chip are fabricated separately on different wafers and assembled afterward. The mirror chip is made out of a silicon on insulator (SOI) wafer. The $10 \mu \mathrm{m}$ thick silicon on insulator layer (or, device layer) is structured into (horizontal) mirrors and frame by bulk micromachining. The optical active side of the mirror is the backside of the device layer, which must be released during fabrication. Intrinsically the device layer backside is optically flat in terms of roughness and, when released, optical flat in terms of planarity (better than $\lambda / 20$ ) over a rectangle of $100 \times 200 \mu \mathrm{m}^{2}$. The suspension structure and the landing posts are realized by surface micromachining of a deposited and doped polycrystalline silicon layer underneath the mirror and frame. Poly-silicon is used rather than another material as it has a thermal expansion coefficient similar to single crystal silicon. This is important for the operation in cryogenic environment. In order to assure the thermal expansion compatibility with the mirror chip, the electrode chip is also based on a SOI silicon wafer. Beside the electrodes, connecting lines and connecting pads, the electrode chip also contains the spacer elements, which ensure a constant gap between the electrode and the mirror chip. The spacer height is fixed and defined by the thickness of the device layer of the electrode chip, therefore the uniformity of the spacer height (and the uniformity of tilt-angle) depends on the uniformity in thickness of this silicon layer. The effective gap height can be tuned during the etching of the electrode.

\subsection{Modeling and simulation}

Tilt angle and actuation voltage are a function of the suspension and actuator design. In this section we discuss the effects of the suspension geometry and electrode position. We consider the case of a cantilever suspension as shown in Figure 2. Assuming a thin (i.e. width $\gg$ thickness) cantilever we can neglect the in-plane movement of the mirror and can consider only the movement in the plane perpendicular to the mirror. Furthermore if we 
consider that the mirror is much thicker and larger than the cantilever, we can assume the mirror to be rigid *. The electrostatic forces acting on the mirror can then be reduced to a resulting force and moment acting on the point where the cantilever is attached to the mirror, as shown in Figure 2. The resulting piston movement and tilt angle due to the force $F$ and the moment $M$ is obtained by linear superposition, i.e. summation of the two individual contributions. Considering small deflections we can write for the $\mathrm{y}$-deflection of the cantilevers end

$$
\begin{aligned}
\delta & =\delta_{F}-\delta_{M} \\
& =\frac{l_{c}^{2}}{E I_{y}}\left(\frac{F l_{c}}{3}-\frac{M}{2}\right)
\end{aligned}
$$

and for the angle

$$
\begin{aligned}
\alpha & =\alpha_{F}-\alpha_{M} \\
& =\frac{l_{c}}{E I_{y}}\left(\frac{F l_{c}}{2}-M\right)
\end{aligned}
$$

where $l_{c}$ is the cantilever length, $E$ Young's modulus and $I_{y}$ the moment of inertia around the y-axis. ${ }^{5}$ The moment of inertia is given with $I_{y}=w d^{3} / 12$, where $w$ is the width of the cantilever and $d$ the thickness. Note that equation 1 represents the (vertical) piston movement of the mirror and equation 2 the tilt angle. It is obvious from equation 2 that for a mirror movement as shown in Figure 2, we must have $F l_{c}>2 * M$. If we have $F l_{c}<2 * M$ the mirror tilts in the opposite direction. We assume a constant electric field between the mirror and the electrode, and thus a constant force intensity $F / l_{m}$ acts on the mirror. The resulting force would then be $F$ and the resulting moment

$$
\begin{aligned}
M & =\int_{-b}^{0} \frac{F}{l_{m}} x d x+\int_{0}^{l_{m}} \frac{F}{l_{m}} x d x \\
& =\frac{l-2 b}{2} F
\end{aligned}
$$

where $b$ denotes the position of the attachment point of the cantilever to the mirror, measured from the left mirror edge (see Figure 2). By combining equations 1- 3, we can predict the mirror angle and piston in function of the cantilever attachment offset $b$.

This simple considerations give a rough idea about the mirror tilting behavior in function of the suspension geometry. Considering the real case, where large deflections and large angles occur, simulations using the finite element method (FEM) must be carried out. Large out-of-plane movements, especially tilting movements, often cause convergence problems in coupled electrostatic and mechanical simulation due to strong mesh deformation and non-linearities. Non-linearities occur due to stress-stiffening, which must be taken into account when considering large deflections of the cantilever and can not be avoided. Mesh-deformation can be avoided by re-meshing the electrostatic and mechanical model after each iteration. Thus we developed a script-based custom 2D electromechanical model for the use with ANSYS. The principal idea is to separate the mechanical and electrostatic model. The electrostatic model calculates the forces that act on the mirror; the resulting force and moment is then transferred onto the cantilever in the mechanical model. The simulation of the mechanical model gives then the deflection of the cantilever and the new position of the mirror, which is again transferred into the electrostatic model. For each iteration the electrostatic model is rebuild and re-meshed based on the geometrical data calculated by the mechanical (cantilever) model. Simulations where carried out varying the key-parameters of the micromirror device: cantilever geometry, cantilever position, electrode position and gap height. Tilt angle, pull-in voltage, maximum stress and the first resonance frequency were extracted from the simulations. The first resonance frequency, which is a measure for shock resistivity, is between $800 \mathrm{~Hz}$ and $2 \mathrm{kHz}$ for different designs, which is an acceptable range for the considered application. The maximum stress in the cantilever, ranges from $120 \mathrm{MPa}$ to $400 \mathrm{MPa}$, designs which exceeds $400 \mathrm{MPa}$ are not considered.

${ }^{*}$ The deflection $\delta$ due to a force $F$ of a beam with rectangular section $w h$ can be written as $\delta \sim F / w h^{3}$. Assuming a beam with 10x0.5 $\mu \mathrm{m}$ section and a mirror with a 200x10 $\mu \mathrm{m}$ section, having the same length, a force $F$ would deflect the mirror $20^{4}=160000$ times less than the cantilever. 

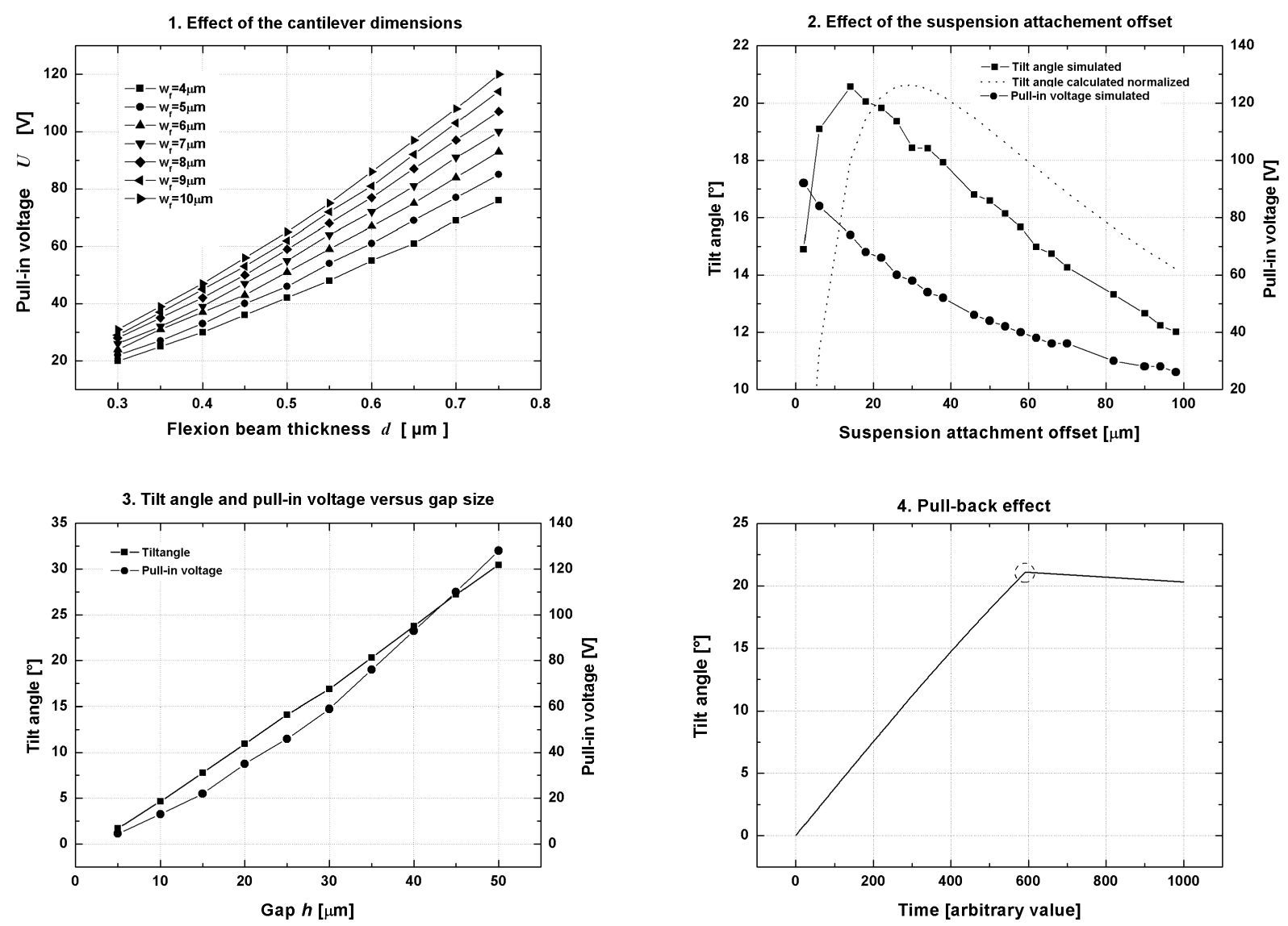

Figure 4. Simulation results. 1. Pull-in voltage versus flexion beam thickness for different flexion beam widths for a gap height $h=35 \mu \mathrm{m}$ and a suspension offset $b=20 \mu \mathrm{m}$. 2. Tilt angle and pull-in voltage versus suspension offset $b$ for a gap height $h=35 \mu \mathrm{m}$ and flexion beam dimensions $d=0.5 \mu \mathrm{m}$ and $w=10 \mu \mathrm{m}$. As comparison, a normalized plot of the analytic formula for the pull-in voltage is drawn (dashed curve). 3. Tilt-angle and pull-in voltage versus gap height $h$ for $b=20 \mu \mathrm{m}$ and $d=0.55 \mu \mathrm{m}$. The linearity between tilt-angle and gap height can clearly be seen. 4 . Evolution of the tilt angle for $h=35 \mu \mathrm{m}$ and $b=20 \mu \mathrm{m}$, demonstrating the pull-back effect: after the landing posts on the mirror touch the electrode (circled in the graph), the mirror starts to rotate in the inverse direction and the tilt-angle decreases again. Together with suitable system of landing posts, this effect should yield a constant tilt-angle. 
Figure 4 shows a selection of simulation data, considering tilt angle and actuation voltage. For a given gap height the pull-in voltage depends strongly on the dimensions of the cantilever. The first graph shows the pull-in voltage in function of the thickness and the width of the cantilever. The length of the cantilever is $100 \mu \mathrm{m}$ and the gap height is $35 \mu \mathrm{m}$. We remark a strong dependence on the thickness of the cantilever $d_{f}$, which corresponds to the first order analytical formula which predicts $U \sim d_{f}^{3 / 2}$.

The position of attachment of the cantilever to the mirror $b$ has also a strong influence on the pull-in voltage and tilt angle for a given gap height. We are looking for the tilt angle, normalized with the gap height, in function of the parameter $b$. The gap height equals $\delta+b \alpha$ for small angles. Assuming $l_{c}=l_{m} \equiv l$ we get

$$
\frac{\alpha}{\delta+\alpha b}=\frac{b}{b^{2}+\frac{l}{2} b+\frac{l^{2}}{12}}
$$

The function is plotted together with the simulation results in the second graph of Figure 4. The simulation was carried out for a cantilever suspension with the dimensions $0.5 \times 10 \times 100 \mu \mathrm{m}^{3}$ and a gap height of $35 \mu \mathrm{m}$. The function plot is normalized to the maximum value of the simulated curve. Note that the maximum for the simulated curve occurs at about $b=15 \mu \mathrm{m}$ and the maximum of the calculated curve at $b=30 \mu \mathrm{m}$. This difference is due to the shifted electrode (parameter $e$, see Figure 2) used for the simulation. In the calculation we have used $e=0$ and for the simulation $e=10 \mu \mathrm{m}$. Indeed we found by simulation that in terms of actuation voltage and tilt angle the best value for $e$ is between $10 \mu \mathrm{m}$ and $20 \mu \mathrm{m}$, depending on the suspension offset $b$. The pull-in voltage curve is monotonic decreasing, while the tilt angle curve first increases to reach a maximum around $b=15 \mu \mathrm{m}$ and then decreases monotonic. When normalizing the tilt angle with the pull-in voltage, the curve is monotonic increasing, that is for an increasing $b$ we get an increasing ratio tilt angle per volt. If the lowest pull-in voltage for a given tilt angle is searched, then a big $b$ must be chosen, i.e. the cantilever must be attached to the right side of the mirror in Figure 2. The result is a large piston movement of the mirror toward the electrode, which increases the shadowing of the mirror. Therefore, as long as the actuation voltage is under $100 \mathrm{~V}$, we are rather interested in having as much as tilt angle per gap as possible. In this case the trade-off value for $b$ is between $10 \mu \mathrm{m}$ and $20 \mu \mathrm{m}$.

In the third graph the dependence of snap-in voltage and tilt angle on the gap height $h$ is shown. The simulation was carried out for a cantilever suspension with the dimensions $0.55 \times 10 \times 100 \mu \mathrm{m}^{3}$ with $b=20 \mu \mathrm{m}$. The relation between tilt angle and gap height is linear, what can be expected from a geometrical point of view. The snap-in voltage versus gap height relation is of the form $\sim x^{a}$ with $a>1$. We extract from this simulation a required gap height of $35 \mu \mathrm{m}$ for an tilt angle of $21^{\circ}$ resulting in a pull-in voltage of $78 \mathrm{~V}$.

Finally, in order to demonstrate the pull-back effect exhibited in the previous subsection, the evolution of the tilt angle before it reaches the 'ON' state is shown in the fourth graph. The tilt angle is monotonically increasing until a certain point, where the mirror (or, the landing post on the mirror) hits the electrode. After this point the tilt angle decreases again until it reaches its equilibrium position. If we introduce a landing post in such way that the mirror is blocked for a tilt angle between the maximum and equilibrium value, the mirror is electrostatically clamped in this position, which is independent from voltage and process variations.

\section{REALIZATION}

Arrays of $2 \times 2$ and $5 \times 5$, as well as single mirrors with either torsion or suspension beam suspension and different types of electrodes have been fabricated. Mirror sizes of 100x200 $\mu \mathrm{m}^{2}, 200 \mathrm{x} 100 \mu \mathrm{m}^{2}$ and 250x500 $\mu \mathrm{m}^{2}$ have been implemented. Flexion and torsion beams with various lengths and widths and a thickness of $0.6 \mu \mathrm{m}$ have been realized. The fabrication includes the processing of the mirror wafer, the processing of the electrode wafer and the assembly of the released mirror chips and diced electrode chips.

Figure $5 \mathrm{a}$ )-e) shows the fabrication process of the mirror chip. The $10 \mu \mathrm{m}$ thick device layer of an SOI wafer is structured into mirrors and frame by deep reactive ion etching (DRIE). At the same time the trenches for the dice free release ${ }^{6}$ have been defined. During the next step $2.2 \mu \mathrm{m}$ thermal wet silicon dioxide is grown in order to fill the trenches between the mirrors and the frame. Reactive ion etching (RIE) is used afterward to open the $\mathrm{SiO}_{2}$ where the suspension is attached to the mirrors and the frame. A poly-silicon layer is then deposited by chemical vapor deposition (CVD) and doped. Then the suspension and the landing posts are structured into the 
a)
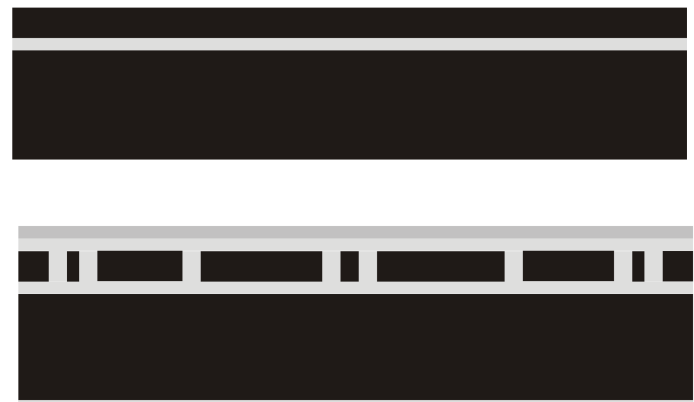

b)

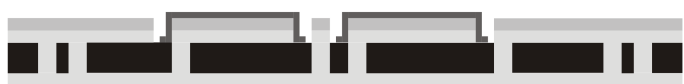

c)

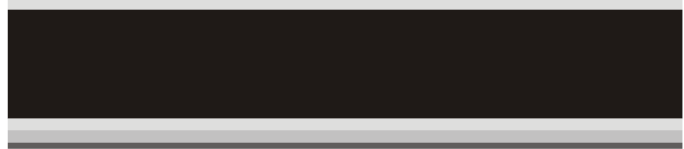

d)

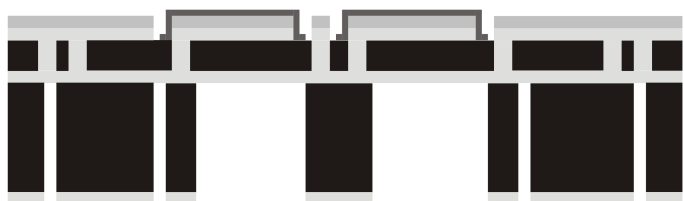

e)

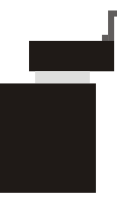

f)

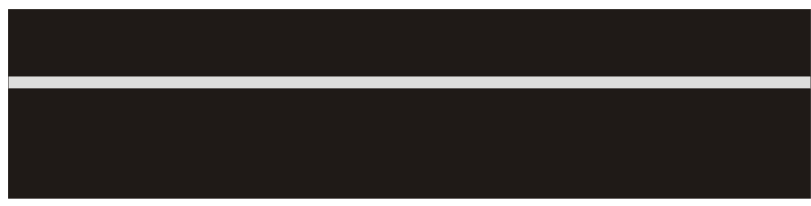

g)
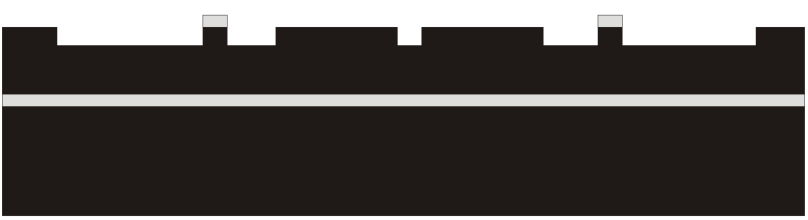

h)

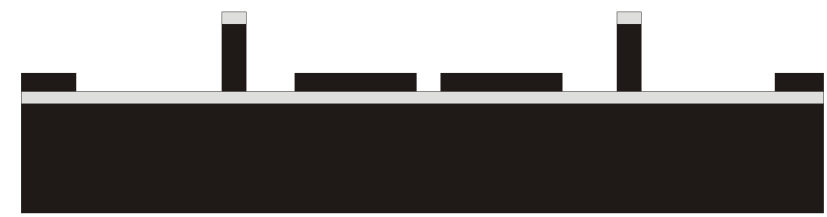

i)

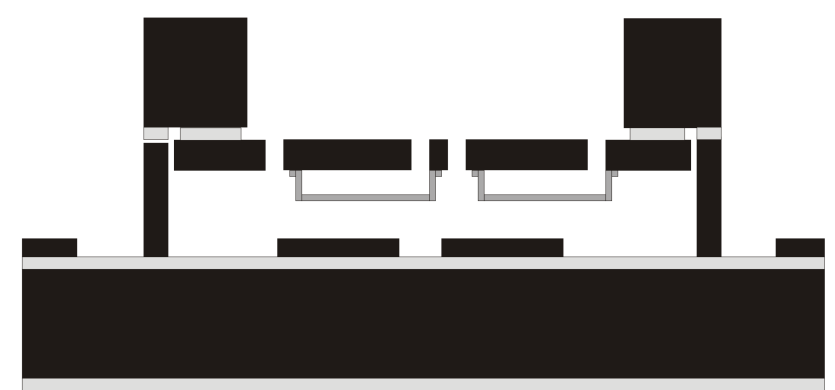

Figure 5. Process flow. Mirror chip: a) A SOI wafer with a $10 \mu \mathrm{m}$ thick device layer is used as substrate b) Mirrors and frame are defined by DRIE, the gaps are filled by oxidation c) The oxide is opened by RIE at the points of attachment of the suspension and the landing posts, deposition by CVD and structuring by DRIE of the poly-silicon layer d) Opening of the mirrors by backside DRIE e) HF vapor release of the mirrors and the chips. Electrode chip: f) A SOI wafer with a $50 \mu \mathrm{m}$ thick device layer is used as substrate g) Definition of spacers (protected by an oxide mask) and electrodes by a first DRIE step h) Transfer of the electrode pattern to the bottom of the device layer by a second DRIE step. Assembly: h) The mirror chip is put on the spacers of the electrode chip and aligned. 

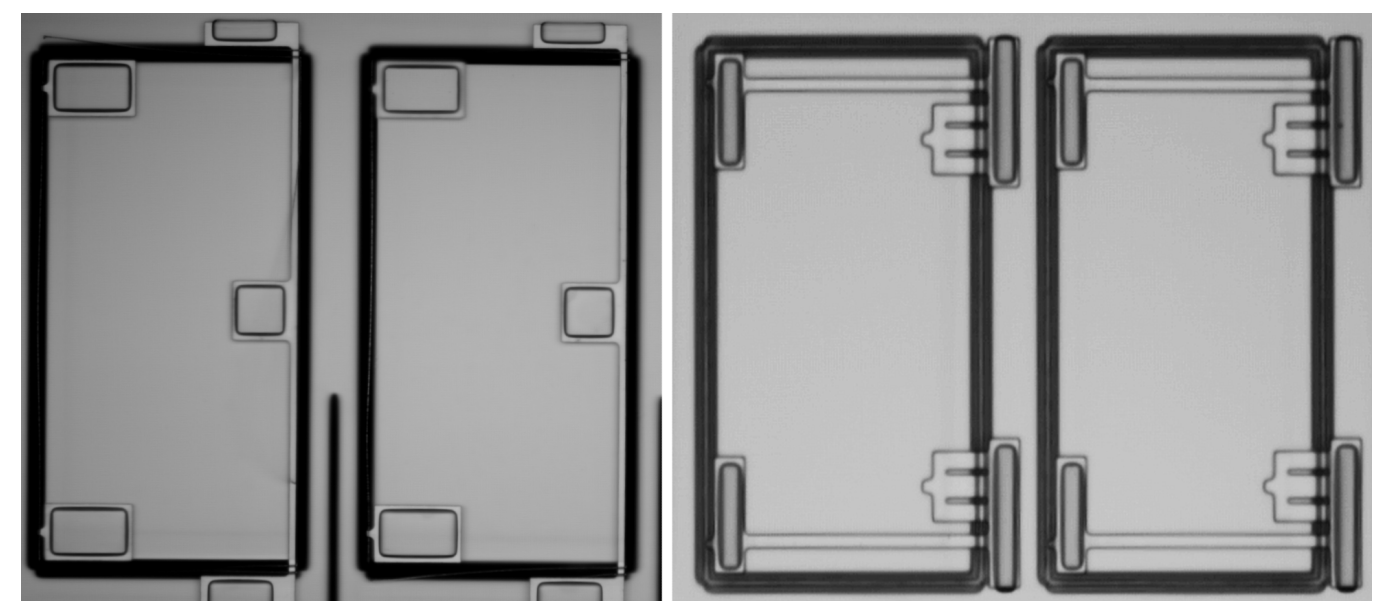

Figure 6. Micrographs of fabricated mirrors after the final release step. Left: Micrograph of single mirrors with torsion beam suspension. Right: Flexion beam suspension. The extra pads attached to the frame at the mirrors right side will be used for the electrostatic clamping mechanism

poly-silicon layer. DRIE has been used, in order to preserve the dimensions of the fine poly-silicon structures to a maximum extend. In a final DRIE step the backside openings of the mirror and the dice free chip release trenches are etched into the $350 \mu \mathrm{m}$ handle layer. First the mirrors and then the whole chips are released in a dry HF vapor etch step. ${ }^{6}$ The mirror chips are now ready for assembly with the electrode chip. Figure 6 shows micrographs of released single mirrors with torsion and flexion suspension. The landing posts attached to the frame in the case of the cantilever suspension are visible on the right side of each micro-element. The landing posts attached to the mirror are located on the left side of each mirror. The torsion beam does not show any buckling, which could have resulted in an in-plane rotation of the mirror. The faint bright line visible in the gap between the mirror and the frame is a poly-silicon residue. A process modification which should prevent the formation of these poly-silicon residues is under way.

Figure $5 \mathrm{f}$ )-h) shows the fabrication process of the electrode chip. A SOI wafer with a $50 \mu \mathrm{m}$ thick device layer is patterned using a self-aligned delay mask process. ${ }^{7}$ In the first step a $0.5 \mu \mathrm{m}$ thick thermal silicon dioxide is grown. In the first photolithography and subsequent RIE step the spacer mask is coarsely defined in the oxide mask. In the second photolithography and RIE step the precise form of the spacer is defined in the oxide mask and at the same time the electrodes, connection pads and connecting lines are patterned into photoresist. Then by time controlled deep reactive ion etching the first couples of micrometers are etched. This step defines the height of the electrodes and connecting lines. After an oxygen plasma resist strip the remaining thickness of the device layer is etched. In that way the electrode and connecting lines pattern is transferred to the bottom of the device layer, while the spacers, protected by an silicon dioxide mask, still have the initial height of the device layer. In a final step the wafer is diced to obtain the individual electrode chips. Figure 7 shows a micrograph of the electrode chip ready for assembly. The electrodes of the first run showed heights ranging from $4 \mu \mathrm{m}$ to $15 \mu \mathrm{m}$ and the spacers a height of $48 \mu \mathrm{m}$. Within one chip the variation of the electrode height is smaller than $100 \mathrm{~nm}$ and the variation of the spacer height smaller than $10 \mathrm{~nm}$.

The last fabrication step is the assembly of the electrode and the mirror chip (Figure 5i)). The mirror chip is placed upside down on the angled quad spacers of the electrode. The mirror chip is then pushed parallel to the angled squads (in Figure 7 this would be upwards). The angled squads with their counterparts on the mirror chip guide the mirror chip to the good position. Once the electrodes and mirrors are aligned, a clipping system on the mirror chip snaps in and holds the mirror in the aligned position. The clipping system holds the device together under moderate accelerations, but for durable assembly the chips will be fixed with epoxy glue. In Figure 7 an assembled device is shown. The dimensions of the device are $5 \times 5 \mathrm{~mm}^{2}$. It contains one $5 \times 5$ and two $2 \times 2$ arrays and three test fields, each of them containing six single mirrors. Different values for the suspension and landing posts dimensions have been implemented in the test field. The effective gap between the mirror and 


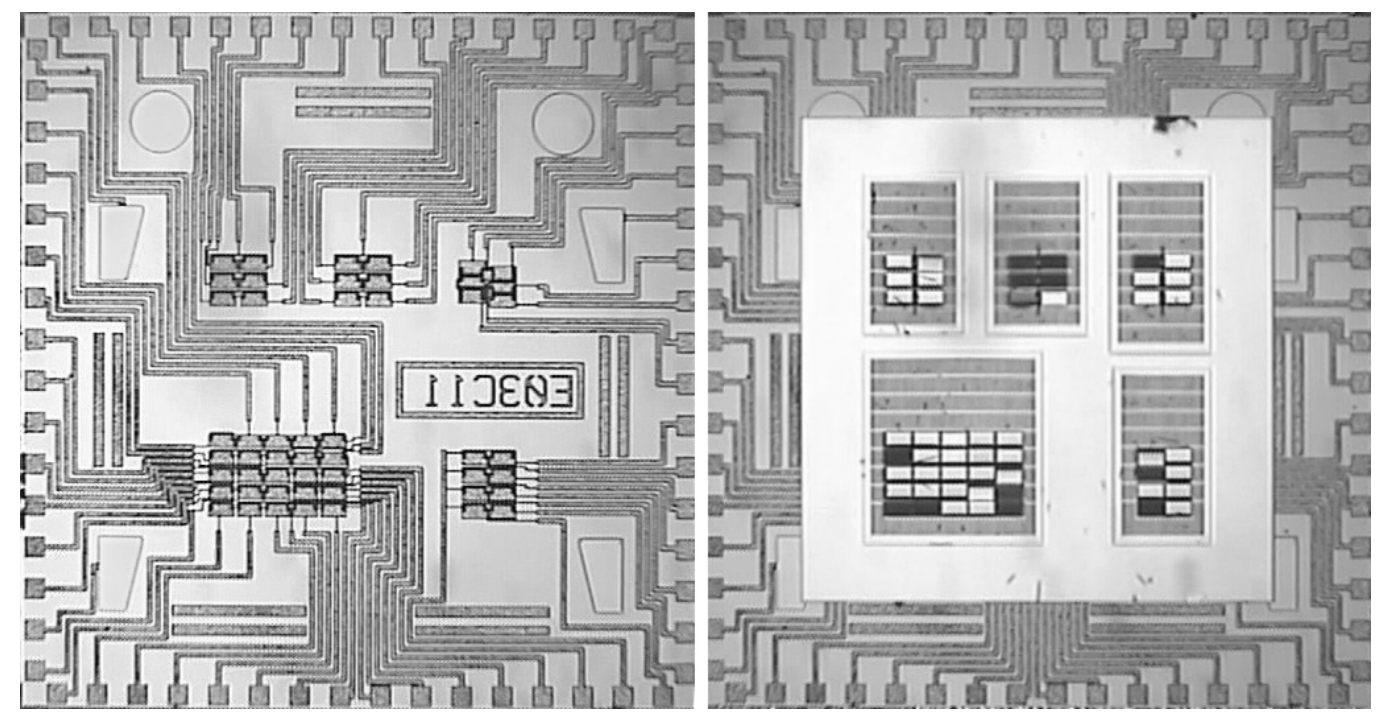

Figure 7. Micrograph of the fabricated electrode (left) and the assembled device (right). The mirror chip is put upside down on the angled quads located around the electrode field on the electrode chip. The device features a $5 \times 5$ (bottom left) and two 2x2 (bottom right) arrays, as well as three test fields. The mirrors that appear black are slightly tilted, the others are non-tilted.

the electrode ranges, for different electrode chips, from $20 \mu \mathrm{m}$ to $36 \mu \mathrm{m}$.

\section{CHARACTERIZATION}

\subsection{Mechanical and electrical characterization}

Fabrication yielded in over $95 \%$ of functional mirrors and less than $5 \%$ of the mirrors had a broken suspension or have been torn off completely. The critical step, i.e. where damage to the suspension can occur, is the release step and before all, the assembly. Since the suspension extends out of the mirror plane, it is quite sensitive to mechanical contact, for instance if the mirror chip is laid upside down on a surface other than the electrode chip. The suspension shows to be mechanical stable and reliable for the designed tilt-angle - the stress occuring in the flexion or torsion beams when tilted $20^{\circ}$ is much lower than the rupture point; $90^{\circ}$ tilting (by vacuum actuation) has been obtained in a repetitive manner without damaging the suspension. No buckling and no sticking of the torsion or flexion beam was observed and the mirrors are not rotated in plane due to a stressed suspension. No lateral sticking of the mirrors has been observed. A slight initial tilting of different mirrors can be seen in most of the devices (see Figure 7). This may be due to a slightly stressed polysilicon-silicon interface, which could cause the suspension beam to bend slightly. Another contribution could come from the parasitic poly-silicon nanowires and static charging, when the mirrors are left on a floating potential.

Preliminary tests shows that the mirrors with both, the torsion and flexion beam suspension, can be actuated as predicted. The snap-in voltage is in the range of $50 \mathrm{~V}$ to $110 \mathrm{~V}$ for the different designs and different gap sizes, which corresponds to the order of magnitude given by the simulations. The mirrors with the torsion beam suspension show the typical voltage-tilt angle hysteresis. Once the mirror is actuated with the pull-in voltage it stays in the 'ON' position; when lowering the actuation voltage below approximatively half of the snap-in voltage the mirror starts to return in its 'OFF' position. Figure 8 shows a sequence of micrographs of an actuated single mirror with cantilever suspension. For test purposes here the suspension is lateral to the frame, which allows characterization of the cantilever bending. In the first image the applied voltage is $0 \mathrm{~V}$ and the mirrors are all in the 'OFF' position. Then an intermediate voltage of $30 \mathrm{~V}$ is applied to the electrode underneath the mirror on the bottom right and the mirror starts to tilt and stays in an intermediate position. When the voltage is further increased, the mirror tilts more and more until it snaps into the 'ON' position, when the voltage reaches the pull-in value (here $75 \mathrm{~V}$ ). Note that during this operation all the other mirrors remained in the 'OFF' position, 

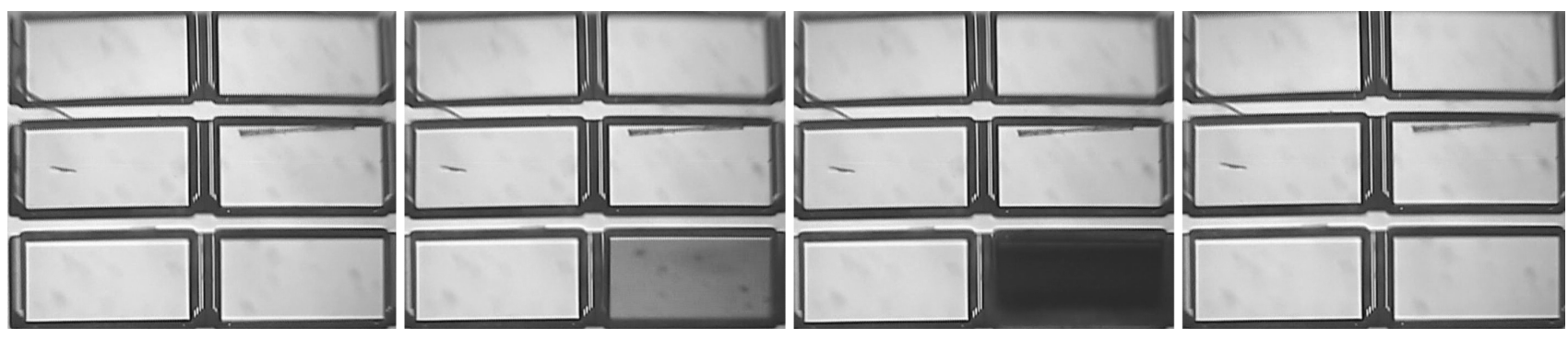

Figure 8. Sequence of micrographs illustrating the actuation of one mirror out of six. The cantilever suspension here is lateral to the mirror for characterization purposes. The pull-in voltage (third image) is $75 \mathrm{~V}$. Note that visibly there is no crosstalk between the mirrors.

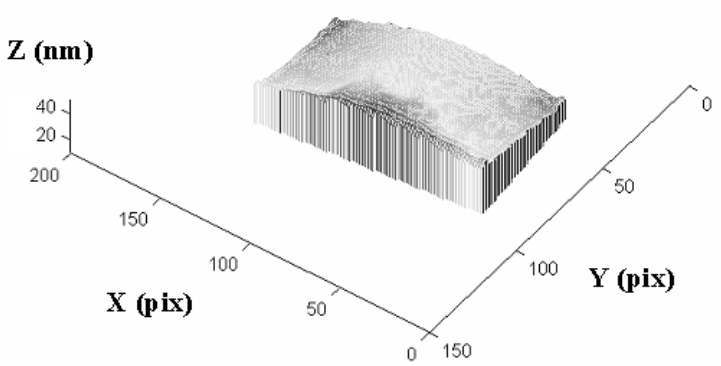

Figure 9. Quality measurement of an individual micro-mirror $100 \mu \mathrm{m} \times 200 \mu \mathrm{m}$ (1 pixel $=2 \mu \mathrm{m}), 3 \mathrm{D}$ view. The peak-tovalley deformation is $30 \mathrm{~nm}$

i.e. no crosstalk was observed. When resetting the voltage to $0 \mathrm{~V}$ the mirror snaps back into the initial 'OFF' position. The leakage current between the mirror and the electrode during the whole operation was negligible.

\subsection{Optical characterization}

Due to its location at the focal plane of the spectrograph, the surface quality of each micro-mirror must be very high, i. e. better than $\lambda / 20$. In the 'ON' position, any surface aberration will result in an image quality degradation on the detector of the spectrograph. In addition, all micro-mirrors must tilt by the same angle for optimizing the optical design of the instrument; if there is a variation in this angle, all optics have to be oversized for keeping the required performances. In the 'OFF' position, the requirement on the mirror location is less accurate, as these mirrors send the light back toward the telescope. The mirror surface must also remain flat in operation throughout a large temperature range. At LAM, a dedicated characterization bench has been developed for the complete analysis of MOEMS devices, actuators or micro-mirrors as well as full arrays. This modular Twyman-Green interferometer allows high in-plane resolution $(4 \mu \mathrm{m})$ or large field of view $(40 \mathrm{~mm})$. Out-of-plane measurements are performed with phase-shifting interferometry showing very high resolution (standard deviation $<1 \mathrm{~nm}$ ). Features such as optical quality or electro-mechanical behavior are extracted from these high precision three-dimensional component maps. Range is increased without loosing accuracy by using two-wavelength phase-shifting interferometry authorizing large steps measurements. Dynamic analysis like vibration mode and cut-off frequency is also measured with time-averaged interferometer. ${ }^{8}$

Preliminary characterization is done on a $5 \times 5$ array made of $100 \mu \mathrm{m}$ x $200 \mu \mathrm{m}$ micro-mirrors. The device is not actuated and the mirrors are in the 'OFF' position (mirrors parallel to the frame / substrate). This sample is attached vertically on the bench for characterization. One mirror out of twenty five is aligned with the reference flat mirror of the interferometer and fringes are visible on the other micro-elements. These fringes show 
differential tilt angles in the 'OFF' position. Most of the mirrors are in the same position compared to our test mirror within a range of $0.5^{\circ}$; the most tilted mirror exhibit a maximal angle below $1^{\circ}$. Then, the surface quality of the micro-mirror is measured by phase-shifting interferometry, and a total aberration of $30 \mathrm{~nm}$ peak-to-valley is measured (Figure 9). This value, better than $\lambda / 20$, is within the requirement. This is a very promising result for future operation in a spectrograph, when the surface flatness of the mirror during operation (tilted mirror) will be confirmed.

\section{CONCLUSION AND OUTLOOK}

We have developed and fabricated micromirror arrays as programmable slits for multi object spectrographs (MOS). By using a combination of bulk and surface micromachining on SOI wafers we were able to fabricate arrays up to $5 \times 5$ micromirrors, with a high fill-factor. The typical size of the mirrors is $100 \times 200 \mu \mathrm{m}^{2}$ and the surface measurement shows an optical quality better than $\lambda / 20$ in the 'OFF' state. The electrodes with integrated spacers have been fabricated on a second SOI wafer, using a delay mask process. The assembled device, i.e. mirror chip and electrode chip, is operational and the mirrors are able to tilt by $20^{\circ}$, which is within the requirement for an expected contrast of 3000:1. The actuation voltage is below 100V. These preliminary results including high fill-factor, low actuation voltage, high tilt angle and good optical surface quality are promising for generating reflective slit mask for MOS. Furthermore we have developed, and validated by simulation, a system of landing posts, which should guarantee constant and precise tilt angle over the whole array.

Complete characterization of these prototypes are under way in order to confirm the surface planarity of the mirrors in the actuated location ('ON' state) and in order to validate the function of the landing posts. Improvement of the fabrication process is also under way. Based on these results, we will soon design larger micromirror arrays suitable for implementation in future space and ground-based astronomical instrumentation.

\section{ACKNOWLEDGMENTS}

We gratefully acknowledge the COMLAB staff at the joint microfabrication facility of IMT and CSEM and we are thankful for the financial support of the European OPTICON network.

\section{REFERENCES}

1. F. Zamkotsian and K. Dohlen, "Performance modeling of JWST near infrared multi-object spectrograph," in Proceedings of the SPIE conference on Astronomical Telescopes and Instrumentation 2004, Proc. SPIE 5487, (Glasgow, United Kingdom), 2004.

2. H. Moseley, S. Aslam, M. Baylor, K. Blumenstock, R. Boucarut, A. Erwin, R. Fettig, D. Franz, T. Hadjimichael, J. Hein, A. Kutyrev, D. M. M. Li, C. Monroy, and D. Schwinger, "Microshutter arrays for the NGST NIRSpec," in Proceedings of the SPIE conference on Astronomical Telescopes and Instrumentation 2002, Proc. SPIE 4850, (Hawaii, USA), 2002.

3. F. Zamkotsian, J. Gautier, and P. Lanzoni, "Characterization of MOEMS devices for the instrumentation of next generation space telescope," in Proceedings of the SPIE conference on MOEMS 2003, Proc. SPIE 4980, (San Jose, USA), 2003.

4. W. Noell, P.-A. Clerc, L. Dellmann, B. Guldimann, H. Herzig, O. Manzardo, C. Marxer, K. Weible, R. Dändliker, and N. de Rooij, "Applications of SOI-based optical MEMS," IEEE Journal of Selected Topics in Quantum Electronics Vol. 8, pp. 148-154, 2002.

5. W. C. Young, Roark's Formulas for Stress and Strain, Mc Graw Hill, New York, 8th edition ed., 1989.

6. T. Overstolz, P. A. Clerc, W. Noell, M. Zickar, and N. F. de Rooij, "A clean wafer-scale chip-release process without dicing based on vapor phase etching," in Technical Digest of the 17th IEEE International Conference on Micro Electro Mechanical Systems, pp. 717-720, (Maastricht, The Netherlands), 2004.

7. S. Kwon, V. Milanovic, and L. P. Lee, "Large-displacement vertical microlens scanner with low driving voltage," IEEE Photonics Letters Vol. 14, p. 1572-1574, 2002.

8. A. Liotard, S. Muratet, F. Zamkotsian, and J. Fourniols, "Static and dynamic MOEMS characterization by phase-shifting and time-averaged inteferometry," in Proceedings of the SPIE conference on MOEMS 2005, Proc. SPIE 5716, 2005. 